\title{
Numerical investigation of periodic cavitation shedding in a Venturi
}

\author{
Boris Charrière and Eric Goncalves, B. Charrière ${ }^{1}$, E. Goncalves ${ }^{1}$ \\ ${ }^{a}$ Université Grenoble-Alpes, LEGI, UMR 5519 CNRS, 38041 Grenoble, France \\ ${ }^{b}$ ISAE-ENSMA, institut Pprime, UPR 3346 CNRS, 86961 Chasseneuil, France
}

\begin{abstract}
Unsteady partial cavitation is mainly formed by an attached cavity which present periodic oscillations. Under certain conditions, instabilities are characterized by the formation of vapour clouds convected downstream the cavity and collapsing in higher pressure region. Two main mechanisms have been identified for the break-off cycles. The development of a liquid re-entrant jet is the most common type of instabilities, but more recently, the role of pressure waves created by the cloud collapses has been highlighted. This paper presents one-fluid compressible simulations of a selfsustained oscillating cavitation pocket developing along a Venturi geometry. The mass transfer between phases is driven by a void ratio transport equation model. The importance of traveling pressure waves in the physical mechanism is put in evidence. Moreover, the importance of considering a non-equilibrium state for the vapour phase is exhibited.

Keywords: cavitation, re-entrant jet, pressure waves, 1-fluid simulation, void ratio equation
\end{abstract}

${ }^{*}$ Corresponding author.

Preprint submitted to Elsevier

February 2, 2017 


\section{Nomenclature}

\section{Abbreviation}

CFD Computational Fluid Dynamic

CFL Courant-Friedrichs-Lewy number

DFT Direct Fourier Transform

EOS Equation Of State

KL Smith $k-\ell$ turbulence model

RANS Reynolds-Averaged NavierStokes

RMS Root Mean Square

\section{Greek symbols}

$\alpha$ Void fraction

$\Psi$ Second turbulence variable

$\kappa$ Von Karman constant

$\lambda$ Mixture thermal conductivity

$\lambda_{t}$ Mixture turbulent thermal conductivity $\mu$ Mixture dynamic viscosity

$\mu_{t}$ Mixture dynamic eddy viscosity

$\rho$ Mixture density

$\rho_{l}^{\text {sat }}$ Density of liquid at saturation

$\rho_{v}^{s a t}$ Density of vapour at saturation

$\sigma$ Cavitation parameter

$\overline{\bar{\tau}}$ Mixture total stress tensor

\section{Latin symbols}

$C_{p}$ Heat capacity at constant pressure

$c_{\text {sinus }}$ Minimum speed of sound in the mixture

$e$ Mixture internal energy

$E$ Mixture total energy

$F_{c}$ Convective flux density

$F_{v}$ Viscous flux density

$k$ Mixture turbulent kinetic energy

$\ell$ Integral turbulence length scale

$P$ Mixture pressure

$\operatorname{Pr}$ Prandtl number 
$\operatorname{Pr}_{t}$ Turbulent Prandtl number

$Q$ Mixture total heat flux

Re Reynolds number

$S$ Source term

$S_{i j}$ Stress tensor

$T$ Mixture temperature

$u^{+}$Non dimensional wall velocity

$u_{i}$ Velocity compopent

$U_{\tau}$ Friction velocity

$\vec{V}$ Velocity vector $w$ Vector of conservative variables

$y^{+}$Non dimensional wall distance

\section{Subscript}

inlet Inlet value

$k$ k-phase

ref Reference value

$w$ Wall value

Superscript

$t$ Turbulent part

$v$ Viscous part 


\section{Introduction}

Cavitation is the formation and activity of vapour bubbles or cavities. It may occur through the development or the enlargement of bubbles or cavities present in the liquid. The phenomena is well observed in ship propellers, turbines, pumps and hydrofoils and is often called hydraulic or hydrodynamic cavitation [1]. The cavitation development may be the origin of several negative effects, including noise, vibrations, performance alterations, erosion and structural damages. This makes cavitation an important issue in design and operation, which should be controlled, or at least well understood.

Among the principal types of cavitation that may develop, partial cavitation pockets are often observed in hydraulic machines and is known to be responsible for severe damage. Such cavitating flows can have a complex behaviour where the cavity is characterized by a strong unsteadiness, transient cavities shedding downstream and a completely $3 \mathrm{D}$ flow even in a $2 \mathrm{D}$ configuration. Two regions are generally of interest and are driving the cavitation pattern: the cavity detachment region which is related to the cavitation onset, and the cavity closure where vapour structures formed in the low pressure zones are transported downstream and collapse violently when they reach the higher pressure zone. These shedding of vapour clouds may have different origins. In some cases, the clouds are generated by vortex shedding filled with bubbles or produced by periodic disturbances imposed by the mean flow. For pumps and turbines, these disturbances can be caused by rotor-stator interactions. The experimental and numerical studies identify the presence of two main mechanisms: a re-entrant jet and pressure waves generated by the collapse of larger structures. The re-entrant jet is mainly composed of liquid which penetrates the 
attached cavity from downstream and flows upstream along the solid surface. The motion of a re-entrant jet is central for partial cavitation instabilities but the mechanism which drives the phenomenon remains unclear.

The development of a liquid re-entrant jet was primary described by Knapp et al. [1]. De Lange et al. [2,3] proposed an experimental study of the phenomenon on NACA profiles. Initially, sheet cavity extends on the suction side. When it reaches a sufficient length, the re-entrant jet set up at the closure of the cavity and is directed toward the leading edge. Then, it divides the cavity in a rear part transformed into bubble cloud and convected by the main stream and a front part reduced to a tiny sheet cavity which grows again and starts a new cycle. Kubota et al. [4] measured the velocity of the cloud and showed it was convected with a lower velocity than the bulk flow. Finally, the cloud collapses when it reaches the downstream high pressure area. The same cycle was depicted by Kawanami et al. [5] and they showed that the phenomenon can be aborted by introducing an obstacle along the wall. The jet velocity was measured by Pham et al. [6] and was found to be of the same order of magnitude as the free-stream. Callenaere et al. [7] have found, using LDV measurements, that the re-entrant jet instability occurs when two conditions are satisfied: first, the adverse pressure gradient must be large enough, which imposes a maximum cavity length, second, the cavity must be not too thin, which imposes a minimum cavity length.

The collapse of a shed bubble cloud is known to create pressure waves, often orders of magnitude greater than the pressure in the mean flow. Reisman et al. [8] suggested the presence of shock waves as cloud collapse mechanism, which causes rapid change in the void fraction distribution. Arndt et al. [9] showed for a hydrofoil 
geometry that two competitive mechanisms can be responsible for cloud cavitation. From experimental measurements coupled with numerical simulations, the study distinguished each mechanism using the quantity $\sigma / 2 \alpha$, where $\sigma$ is the number of cavitation and $\alpha$ the angle of attack. For large values a re-entrant jet was found to dominate whereas for smaller values of bubbly flow shock waves dominated. The transition was found to occur for $\sigma / 2 \alpha \simeq 4$. Leroux et al. [10, 11] has suggested that these pressure pulses may contribute to the motion of the re-entrant jet and the cloud cavitation instability. High-speed visualizations of Saito and Sato [12] and Sato et al. [13] about periodic cloud shedding in a convergent-divergent nozzle suggested that the re-entrant motion was triggered by the collapse of shedding clouds and the propagation of bubble collapse. The propagation speed toward upstream was estimated to be on the order of $200-300 \mathrm{~m} / \mathrm{s}$. Stanley et al. [14, 15] described a complex mechanism causing the instability for periodic cavitation shedding in a cylindrical orifice. Using high-speed visualizations, they identified a combination of a travelling pressure wave generated by the bubble cloud collapse and a translational motion of the re-entrant jet, each with distinctly different velocities. The most recent studies have been proposed on a $8^{\circ}$ Venturi nozzle by Ganesh [16]. Based on Laser Doppler Velocimetry (LDV) and X-ray densitometry measurements, their experimental works identified two mechanisms: a re-circulating flow and condensation shock waves, which is the most dominant for a periodic dynamic.

In this paper, we investigate a self-sustained oscillating cavitation pocket developing on a $8^{\circ}$ Venturi nozzle by using compressible RANS simulations. For this geometry, the re-entrant jet phenomenon was observed and described, both experimentally [17, 18] and numerically $[19,20,21,22,23,24]$. The present study focuses on the traveling waves phenomenon and its role in the periodic cloud shedding. Nu- 
merical simulations are performed using an in-house finite-volume code solving a four-equation system composed by three conservation laws for mixture quantities and a supplementary equation for the volume fraction of vapour. The mass transfer between phases appearing explicitly in the formulation is closed by assuming its proportionality with the mixture velocity divergence $[25,26]$. Validation and analysis are done with experimental measurements (time-averaged void ratio and velocity profiles and shedding frequency). The importance of the pressure waves propagation in the physical mechanism is exhibited. Moreover, the effect of a non-constant vapour density is studied, that is to say the vapour phase can reach a metastable state.

This paper is organized as follows. We present first the governing equations, including the physical models, followed by an overview of the numerical methods adopted. The Venturi configuration and the associated numerical parameters are then presented. Unsteady simulations are described and compared with experimental data. A deeper analysis of the pressure waves phenomenon is proposed by using spatio-temporal correlations. The last part deals with conclusions and future investigations.

\section{Equations and models}

The numerical simulations are carried out using an in-house finite-volume code solving the one-fluid compressible Reynolds averaged Navier-Stokes (RANS) system.

\subsection{The one-fluid compressible RANS equations}

The code is based on the solving of a 4-equation system coupled with transportequation turbulence models. For low Mach number applications, an inviscid precon- 
ditioner is introduced. These equations can be expressed as:

$$
\begin{gathered}
P_{c}^{-1} \frac{\partial w}{\partial t}+\operatorname{div}\left(F_{c}-F_{v}\right)=S \\
w=\left(\begin{array}{c}
\rho \\
\rho \vec{V} \\
\rho E \\
\alpha \\
\rho k \\
\rho \Psi
\end{array}\right) \quad ; \quad F_{c}=\left(\begin{array}{c}
\rho \vec{V} \\
\rho \vec{V} \otimes \vec{V}+P \overline{\bar{I}} \\
(\rho E+P) \vec{V} \\
\alpha \vec{V} \\
\rho k \vec{V} \\
\rho \Psi \vec{V}
\end{array}\right) \quad ; F_{v}=\left(\begin{array}{c}
0 \\
\overline{\overline{\tau^{v}}}+\overline{\overline{\tau^{t}}} \\
\left(\overline{\overline{\tau^{v}}}+\overline{\overline{\tau^{t}}}\right) \cdot V-Q^{v}-Q^{t} \\
0 \\
\left(\mu+\mu_{t} / \sigma_{k}\right) \operatorname{grad} k \\
\left(\mu+\mu_{t} / \sigma_{\Psi}\right) \operatorname{grad} \Psi
\end{array}\right)
\end{gathered}
$$

where $w$ denotes the conservative variables and the void ratio, $F_{c}$ and $F_{v}$ the convective and viscous flux densities and $S$ the source terms, which concern the void ratio equation and the transport equations. The expression of the preconditioning matrix $P_{c}$ is given in [26]. $k$ is the mixture turbulent kinetic energy and $\Psi$ is a mixture turbulent variable.

The exact expression of the eddy-viscosity $\mu_{t}$ and the source terms depends on the turbulence model as well as constants $\sigma_{k}$ and $\sigma_{\Psi}$.

The total stress tensor $\overline{\bar{\tau}}$ is evaluated using the Stokes hypothesis, Newton's law and the Boussinesq assumption. The total heat flux vector $Q$ is obtained from the Fourier law involving a turbulent thermal conductivity $\lambda_{t}$ with the constant Prandtl number hypothesis.

$$
\begin{aligned}
\overline{\bar{\tau}} & =\overline{\overline{\tau^{v}}}+\overline{\overline{\tau^{t}}}=\left(\mu+\mu_{t}\right)\left[\left(\operatorname{grad} \vec{V}+(\operatorname{grad} \vec{V})^{t}\right)-\frac{2}{3}(\operatorname{div} \vec{V}) \overline{\bar{I}}\right] \\
Q & =Q^{v}+Q^{t}=-\left(\lambda+\lambda_{t}\right) \operatorname{grad} T \quad \text { with } \quad \lambda_{t}=\frac{\mu_{t} C_{p}}{P_{r t}}
\end{aligned}
$$

In pure phases, the viscosity is assumed to be constant. The mixture viscosity is 
defined as the arithmetic mean of the liquid and vapour viscosities:

$$
\mu(\alpha)=\alpha \mu_{v}+(1-\alpha) \mu_{l}
$$

The mixture thermal conductivity $\lambda$ is also defined as the arithmetic mean of the liquid and vapour values:

$$
\lambda(\alpha)=\alpha \frac{\mu_{v} C_{p_{v}}}{P_{r_{v}}}+(1-\alpha) \frac{\mu_{l} C_{p_{l}}}{P_{r_{l}}}
$$

The turbulent Prandtl number $P_{r t}$ is set to 1.

To compute the pressure and the temperature, an equation of state (EOS) is necessary to link these thermodynamic quantities to the internal energy and the density. For the pure phases, we used the convex stiffened gas EOS:

$$
P(\rho, e)=(\gamma-1) \rho(e-q)-\gamma P_{\infty}
$$

where $\gamma=C_{p} / C_{v}$ is the heat capacity ratio, $C_{p}$ and $C_{v}$ are thermal capacities, $q$ the energy of the fluid at a given reference state and $P_{\infty}$ is a constant reference pressure.

\subsection{The cavitation model}

The cavitation model applied in the present work is based on a void ratio transport equation model:

$$
\frac{\partial \alpha}{\partial t}+\operatorname{div}(\alpha \vec{V})=\left(\frac{\rho_{l} c_{l}^{2}-\rho_{v} c_{v}^{2}}{\frac{\rho_{l} c_{l}^{2}}{1-\alpha}+\frac{\rho_{v} c_{v}^{2}}{\alpha}}+\alpha\right) \operatorname{div} \vec{V}+\left(\frac{\frac{c_{v}^{2}}{\alpha}+\frac{c_{l}^{2}}{1-\alpha}}{\frac{\rho_{l} c_{l}^{2}}{1-\alpha}+\frac{\rho_{v} c_{v}^{2}}{\alpha}}\right) \dot{m}
$$

where $\dot{m}$ is the mass transfer between phases, $\rho_{k}, c_{k}$ are the pure phase density and speed of sound, respectively. 
By assuming that the mass transfer is proportional to the divergence of the velocity, it is possible to build a family of models in which the mass transfer $\dot{m}$ is expressed as $[25,26]$

$$
\dot{m}=\frac{\rho_{l} \rho_{v}}{\rho_{l}-\rho_{v}}\left(1-\frac{c^{2}}{c_{\text {wallis }}^{2}}\right) \operatorname{div} \vec{V}
$$

where $c_{\text {wallis }}$ is the propagation velocity of acoustic waves without mass transfer [27]. This speed of sound is expressed as a weighted harmonic mean of speeds of sound of each phase:

$$
\frac{1}{\rho c_{w a l l i s}^{2}}=\frac{\alpha}{\rho_{v} c_{v}^{2}}+\frac{1-\alpha}{\rho_{l} c_{l}^{2}}
$$

A first model was built using the speed of sound associated with a sinusoidal EOS $[28,29]$ and a second one with a mixture of stiffened gas EOS [30]. These EOS allow to determine the pressure in the mixture. The formulation for the sinusoidal EOS deduces the pressure from the void ratio:

$$
P(\alpha)=P_{\text {vap }}\left(T_{r e f}\right)+\left(\frac{\rho_{l}^{s a t}-\rho_{v}^{s a t}}{2}\right) c_{\text {sinus }}^{2} \operatorname{Arcsin}(1-2 \alpha)
$$

while the stiffened gas EOS proposes the following expression:

$$
\begin{aligned}
P(\rho, e, \alpha, Y) & =(\gamma(\alpha)-1) \rho(e-q(Y))-\gamma(\alpha) P_{\infty}(\alpha) \\
\frac{1}{\gamma(\alpha)-1} & =\frac{\alpha}{\gamma_{v}-1}+\frac{1-\alpha}{\gamma_{l}-1} \\
q(Y) & =Y q_{v}+(1-Y) q_{l} \\
P_{\infty}(\alpha) & =\frac{\gamma(\alpha)-1}{\gamma(\alpha)}\left[\alpha \frac{\gamma_{v}}{\gamma_{v}-1} P_{\infty v}+(1-\alpha) \frac{\gamma_{l}}{\gamma_{l}-1} P_{\infty l}\right]
\end{aligned}
$$

where $c_{\text {sinus }}$ is a parameter of the sinusoidal law, which refers to the minimal speed of sound in the mixture and $Y=\alpha \rho_{v} / \rho$ is the vapour mass fraction.

\subsection{The turbulence modelling}

The two-equation $k-\ell$ model proposed by Smith [31] is used in the present study. Such transport-equation turbulence models always leads to the generation of stable 
cavities, because strong turbulent eddy viscosity $\mu_{t}$ inside the cavity avoids the reentrant jet formation. As a remedy to reduce the turbulent viscosity, we use an ad hoc limiter in the mixture area proposed by Reboud $[32,33]$. This limiter introduces a function $f(\rho)$ in the computation of the turbulent viscosity:

$$
\mu_{t}=f(\rho) \nu_{t} \quad \text { with } f(\rho)=\rho_{v}+(1-\alpha)^{N}\left(\rho_{l}-\rho_{v}\right)
$$

where $N$ is a tunable parameter fixed to 10 .

\subsection{Wall functions}

At the wall, a two-layer wall law is used:

$$
\begin{aligned}
u^{+}=y^{+} & \text {if } y^{+}<11.13 \\
u^{+}=\frac{1}{\kappa} \ln y^{+}+5.25 & \text { if } y^{+}>11.13 \\
u^{+}=\frac{u}{U_{\tau}} ; \quad y^{+}=\frac{y U_{\tau}}{\nu_{w}} \quad ; \quad U_{\tau}=\sqrt{\frac{\tau_{w}}{\rho_{w}}} &
\end{aligned}
$$

where $\kappa=0.41$ is the von Karman constant and the subscript $w$ indicates wall values. We assume that wall functions are similar in a two-phase flow and in a single-phase flow. The validity of this assumption was studied in [34] for Venturi cavitating flows. For unsteady boundary layers, the existence of a wall law is assumed valid at each instant. Comparisons with the thin boundary layer equations were performed and showed the good behaviour of the two-layer approach.

With regard to the turbulent transport-equation models, the production of $k$ is com-

puted according to the formulation proposed by Viegas and Rubesin [35]. The value of $\ell$ in the first cell is computed with the linear relation $\ell=\kappa y$.

\subsubsection{Numerics}

The numerical solving is carried out using an implicit integration based on a finite-volume discretization. For the mean flow, the convective flux density vector 
on a cell face is computed with the Jameson-Schmidt-Turkel scheme [36]. The artificial viscosity includes a second-order dissipation term and a fourth-order dissipation term. The viscous terms are discretized by a second-order space-centered scheme. For the turbulence transport equations, the upwind Roe scheme [37] is used for the convective flux. The second-order accuracy is obtained by introducing a flux-limited dissipation. The diffusive flux is discretized by a second-order centered scheme. Time integration is achieved using the dual time stepping approach and a low-cost implicit method consisting in solving, at each time step, a system of equations arising from the linearization of a fully implicit scheme. The derivative with respect to the physical time is discretized by a second-order formula. The numerical treatment of boundary conditions is based on the use of the preconditioned characteristic relationships. More details are given in $[25,26]$.

\section{Experimental and numerical parameters}

\subsection{Experimental conditions}

The Venturi geometry is characterized by a divergence angle of $8^{\circ}$, illustrated in Fig. 1. The edge forming the throat of the Venturi is used to fix the separation point of the cavitation cavity. This geometry is equipped with eight probing holes (stations 1 to 8 ) to allow various measurements such as the local void ratio, the longitudinal velocity and the wall pressure distribution. The velocity is evaluated as the most probable value and the void ratio is obtained from the signal of the double optical probe using a post-processing algorithm. The relative uncertainty on the void ratio measurement was estimated at roughly $15 \%[18]$.

The selected operating point is characterized by the following physical parameters [18]: 
$U_{\text {inlet }}=7.04 \mathrm{~m} / \mathrm{s}:$ the inlet velocity

$P_{\text {inlet }}=55000 \mathrm{~Pa}:$ the pressure in the inlet section

$\sigma_{\text {inlet }}=\frac{P_{\text {inlet }}-P_{\text {vap }}}{0.5 \rho U_{\text {inlet }}^{2}} \simeq 2.15 \pm 0.06$ : the cavitation parameter in the inlet section $T_{\text {ref }} \simeq 293 \mathrm{~K}$ : the reference temperature

$R e_{L_{r e f}}=\frac{U_{\text {inlet }} L_{r e f}}{\nu}=1.5710^{6}$ : the Reynolds number based on length $L_{r e f}=0.252$ m.

With these parameters, an unsteady cavity with quasi-periodic fluctuations of the attached sheet and vapour cloud shedding has been obtained. The maximum length $L$ of the attached cavity is about $45 \mathrm{~mm}$. The vapour shedding frequency is close to $45 \mathrm{~Hz}$ estimated from Fourier transformations of unsteady pressure signals.

\subsection{Mesh and numerical parameters}

The grid is a H-type topology. It contains 174 nodes in the flow direction and 56 in the orthogonal direction. A special contraction of the mesh is applied in the main flow direction just after the throat to better simulate the two-phase flow area (Fig. 2). The $y^{+}$values of the mesh, at the center of the first cell, vary between 12 and 27 for a non cavitating computation.

Note that only 2D computations are performed here. Nevertheless, it was clearly observed in various experiments that the topology of cavitation pocket in Venturis or hydrofoils is fully three-dimensional, involving a transversal component for the reentrant jet (side-entrant jet). In a previous study [38], 3D simulations of a Venturi geometry were performed with an analysis of the side-wall effects and vortical structures along the corner. Yet, about time-averaged profiles in the mid-span plane, 3D and $2 \mathrm{D}$ simulations provided quite similar results. Relying on these results, only $2 \mathrm{D}$ simulations are considered in this work, also due to the large increase in the compu- 
tational cost.

For the non cavitating regime, computations are started from a uniform flow-field using a local time step. For the unsteady cavitating regime, computations are performed using the dual time stepping method and are started from the cavitating numerical solution. The numerical parameters are:

- the physical time step, $\Delta t=410^{-5} \mathrm{~s}$

- number of sub-iterations for the dual time stepping method, 150

- the CFL number, 0.25

- the farfield value of the turbulent kinetic energy, $k_{\infty}=0.0045 \mathrm{~m}^{2} / \mathrm{s}^{2}$

- the farfield value of the turbulent length scale, $\ell_{\infty}=1.410^{-6} \mathrm{~m}$

\section{Computational results}

Unsteady computations were performed using both EOS formulations. The goal was to obtain a cavitation pocket with time-averaged profiles and oscillation frequency close to experimental values.

\subsection{Comparison of cavitation models}

To evaluate the ability for the 4-equation model to correctly reproduce the cloud cavitation shedding, the sinusoidal law and the stiffened gas EOS are successively used to compute the pressure. The influence of the empirical parameter $c_{\text {sinus }}$ is investigated and three values are tested: $0.47 \mathrm{~m} / \mathrm{s}, 0.92 \mathrm{~m} / \mathrm{s}$ and $1.36 \mathrm{~m} / \mathrm{s}$. For all simulations, the average value of the inlet cavitation parameter is close to 2.1.

A qualitative illustration of cavitation pocket motions is shown in Figure 3 by representing the maximum void ratio on a spatio-temporal graph $(x, t)$. The Venturi 
throat is located at the abscissa $x=0.0012 \mathrm{~m}$. Only $0.5 \mathrm{~s}$ of physical time is presented. We clearly observed the attached cavity evolution (its length is close to 0.01 m) and downstream the quasi periodic cloud shedding. Using the sinusoidal EOS, the break-off cycle frequency increases with the minimal speed of sound value. The cavitating flow behaviour and its periodicity mainly depend on the considered equation of state and, consequently, on the speed of sound formulation.

By assuming that pressure oscillations are mainly created by cloud collapses in the closure region, it is possible to evaluate the phenomenon frequency using a Direct Fourier Transformation (DFT). The obtained frequencies at station 2 (located at $20.9 \mathrm{~mm}$ from the throat) are presented in Figure 4 and show an increase with the minimal speed of sound value. A good agreement with the experimental value is obtained using the highest value $c_{\text {sinus }}=1.36 \mathrm{~m} / \mathrm{s}$. As the mixture speed of sound increases with the value of $c_{\text {sinus }}$, it means that the frequency of cavity destabilization is amplified by the celerity of pressure waves. All results of DFT at stations 1 to 3 are presented in Table 1. The obtained frequencies are quite similar according to the probe location, except for the mixture of stiffened gas EOS for which the frequency decreases (by a factor 1.6) at station 3 .

The previous comparisons highlight the modification of cavitation pocket behaviour by the propagation of pressure disturbances. However, as presented in the introductive part, the re-entrant jet is known as a main responsible in break-off cycles and cloud shedding [18]. In our simulations, this physical mechanism is clearly observed by negative time-averaged velocity profiles near the wall, as illustrated in Figure 5. It is interesting to notice homogeneous values for all computations, which argue that the pressure and the speed of sound determine the periodic shedding frequency without changing the averaged velocity field. However, the void ratio distribution 
depends on the $c_{\text {sinus }}$ parameter: the time-averaged maximum value decreases with the increase of $c_{\text {sinus }}$. This is linked to the different cavity behaviour: when the frequency of pulsations intensifies, a greater number of vapour clouds are convected but their void ratio reduce.

\subsection{Propagation of pressure waves}

A first analysis is based on visualizations of instantaneous pressure and void ratio fields at different times. For the pressure evolution, we use the dimensionless quantity $\left(P-P_{\text {vap }}\right) / P_{\text {vap }}$. Inside the attached pocket or in large-scale cavitation structures, this quantity is close to 0. Visualizations are illustrated in Figure 6 for a total time of $40 \mathrm{~ms}$ corresponding approximatively to 2 cycles. The time interval between two snapshots is $10 \mathrm{~ms}$. Simulations have been performed using the sinusoidal EOS with $c_{\text {sinus }}=1.36 \mathrm{~m} / \mathrm{s}$. On the void ratio field, we clearly observe the attached cavity at the throat and downstream the shedding of different cavitation clouds $N$, $N_{1}, N_{2}$. At the end of the first cycle, the condensation of the cloud $N$ generates pressure fronts which impacts the upstream low-pressure area. This phenomenon is better illustrated in Figure 7 where the time of observation is reduced to $10 \mathrm{~ms}$ and the time interval between two snapshots is around $2 \mathrm{~ms}$. Due to the cloud collapse, the fluid is alternatively compressed and dilated and pressure fronts are generated regularly. By reducing the time interval between 2 snapshots to $0.2 \mathrm{~ms}$, it is now possible to observe in Figure 8 the propagation of one front between times $t_{i}=0.7$ ms and $t_{i}=0.8 \mathrm{~ms}$.

Complementary analysis are proposed using spatio-temporal correlation functions. The correlation function $R_{s}$ quantifies the similitude of signals $s$ evaluated at each time step $\delta t$ between each point $x$ spatially located with a step $\delta x$. It is defined by 
the following relation:

$$
R_{s}(x, \delta x, \delta t)=\frac{\overline{(s(x, t)-\overline{s(x)})(s(x+\delta x, t+\delta t)-\overline{s(x+\delta x})}}{s_{r m s}(x) s_{r m s}(x+\delta x)}
$$

In the present study, we consider 50 points regularly distributed along a line of length $6 \mathrm{~cm}$ located downstream the throat at a distance $2.5 \mathrm{~mm}$ from the wall, as represented in Figure 9. The space step is $\delta x=1.2 \mathrm{~mm}$ and the time step is the one used for simulations $\delta t=410^{-5} \mathrm{~s}$. We compute the correlation function for both the pressure and void ratio signals. Correlations $R_{\alpha}$ give information on the convection velocity of two-phase structures and correlations $R_{p}$ allow to identify slow phenomena associated to the convection of structures and fast phenomena linked to the propagation of pressure waves.

Iso-contours of void ratio and pressure correlations evaluated at stations 2 and 3 are plotted in Figure 10 using only the sinusoidal EOS. Abscissa is the dimensional time. The convection of two-phase structures is clearly highlighted and the associated velocity is estimated to $11 \mathrm{~m} / \mathrm{s}$, that is in the same order of magnitude as the freestream velocity. Moreover, on the $R_{p}$ pressure map, it appears another phenomenon represented by quasi vertical iso-contours, which becomes more intense at station 3 . It can be interpreted as the signature of pressure waves which propagates rapidly in the flow. These correlations are more pronounced downstream station 3 in the region of collapse of clouds, as observed in Figure 11. The evaluation of the slope and therefore the celerity is not easy because the curve is quasi vertical.

We tried to compute the Venturi using a smaller time step of order of a few $\mu$ s. Unfortunately, oscillations of the solution appeared leading to the divergence.

We propose a similar analysis based on simulations performed with $c_{\text {sinus }}=0.47$ $\mathrm{m} / \mathrm{s}$. For this case, the shedding frequency is lower around $24 \mathrm{~Hz}$. Iso-contours of $R_{\alpha}$ and $R_{p}$ are illustrated in Figure 12 at stations 2 and 3. In comparison with 
the previous case, the void ratio correlations put in evidence a slower convection velocity, as expected. An enlargement of the pressure correlations map at station 3 is proposed in Figure 13. We can observe a negative slope for the fast phenomenon illustrated by the black line, that is to say pressure waves propagate toward the throat. The associated velocity is higher than $100 \mathrm{~m} / \mathrm{s}$, corresponding to the Wallis speed of sound with a weak quantity of vapour. This value is lower in comparison with the estimation proposed by Saito and Sato [12] using high-speed video camera on a convergent-divergent nozzle.

\subsection{Saturation versus metastable state for the vapour density}

The void ratio and the mixture density are linked by the relation

$$
\alpha=\frac{\rho-\rho_{l}}{\rho_{v}-\rho_{l}}
$$

For 3-equation models assuming the local thermodynamic equilibrium between phases, densities of pure phases are evaluated at their saturation state function of the local mixture temperature. For isothermal flows (cold water), densities are therefore constant equal to $\rho_{k}^{\text {sat }}\left(T_{r e f}\right)$.

The adding of a void ratio transport equation allows to relax the local thermodynamic equilibrium assumption and to introduce a non-equilibrium (metastable) state for one phase. It is worth noting that metastable states are an integral part of physics of cavitating flows. In our model, we consider that the vapour phase does not remain at its saturation state but evolutes following the stiffened gas EOS

$$
\rho_{v}=\frac{p+p_{\infty v}}{\left(\gamma_{v}-1\right) C v_{v} T}
$$

We propose to compare simulations based on the sinusoidal EOS for the two cases: equilibrium case with the saturation value at the reference temperature $\rho_{v}=\rho_{v}^{\text {sat }}$ 
and metastable case according to the stiffened gas equation of state (the previous results). The value of the parameter $c_{\text {sinus }}$ is set to $1.36 \mathrm{~m} / \mathrm{s}$.

Figure 14 compares the spatio-temporal distribution of maximum void ratio for both cases. The computation performed using the non-constant value involves a more irregular and random behaviour of the cavity. For the saturation case, a stabilization effect is observed: the attached cavity length does not vary over time and the convected structures keep similar shapes.

The previous qualitative observations can be quantified in the closure region. Evolutions of the void ratio, the dimensionless pressure $\left(P-P_{v a p}\right) / P_{v a p}$ and the Mach number at station 2 are plotted in Figure 15 during a physical time of $0.1 \mathrm{~s}$. This time corresponds to approximatively five structures shedding, clearly illustrated by both simulations, with a more irregular behaviour using a metastable vapor density. Between two shedding, in the recompression area, the collapse of structures generates pressure waves. Results clearly highlight that a non-equilibrium state for the vapour phase increases the pressure magnitude. Similar results are observed for the Mach number variations in the shedding region. While the flow remains subsonic for the saturation value, it clearly becomes supersonic when a metastable state is considered. These results question the importance of non-equilibrium effect to reproduce a shock waves propagation caused by the vapour structure collapses.

Discrepancies also appear with statistical analysis. Indeed, in Figure 16, the vapour cloud shedding frequency is reduced using the constant value for the vapour density. It is also the case for Root Mean Square (RMS) wall pressure fluctuations (divided by the time-averaged pressure $P_{a v}$ ) plotted in the Venturi divergent. These 
analysis confirm the initial comment which noticed a more stabilized flow when saturation value is used.

\section{Conclusion}

A periodic self-sustained cavitation pocket developing along a Venturi configuration has been studied by numerical simulations. Calculations have been carried out using a compressible one-fluid RANS code and results have been compared with experimental data concerning the void ratio, streamwise velocity and shedding frequency. Firstly, by comparing two equations of state (a sinusoidal law and a mixture of stiffened gas EOS), it was shown that the break-off cycle frequency depends on the mixture speed of sound estimation. It means that the celerity of pressure waves influences the attached cavity destabilization. Secondly, visualizations of the instantaneous pressure field at different times illustrated pressure fronts due to the collapse of cavitation clouds. Thirdly, the plot of iso-contours of void ratio correlations highlighted the cloud shedding and the convection velocity was found to be of the same order of magnitude as the free-stream velocity. On the other hand, iso-contours of pressure correlations put in evidence a fast phenomenon, which can be interpreted as the propagation of pressure waves. A negative slope was exhibited, that is to say pressure waves can flow up toward the throat.

Finally, the question of considering a non-equilibrium state for the vapour density was discussed. The metastable case involved more irregular and random behaviour of the cavity. For this configuration, Mach number values often exceeded the sonic limit. On the other hand, the saturation case reduced the pressure oscillation frequency and the amplitude of the RMS pressure fluctuations.

Additional works are in progress to pursue comparative analyses and to simulate 3D cavitating flows. 


\section{Acknowledgment}

We thank Direction Générale de l'Armement (DGA) for its financial support to this study. 


\section{References}

[1] R. T. Knapp, J. W. Daily, F. Hammit, Cavitation, McGraw-Hill, New York, 1970.

[2] D. de Lange, G. Bruin, L. van Wijngaarden, On the mechanism of cloud cavitation - experiments and modelling, in: 2nd International Symposium on Cavitation CAV1994, Tokyo, Japan, 1994.

[3] D. de Lange, G. de Bruin, Sheet cavitation and cloud cavitation, re-entrant jet and three-dimensionality, Applied Scientific Research 58 (1998) 91-114.

[4] A. Kubota, H. Kato, H. Yamaguchi, M. Maeda, Unsteady structure measurement of cloud cavitation on a foil section using conditional sampling technique, Journal of Fluids Engineering 111 (2) (1989) 204-210.

[5] Y. Kawanami, H. Kato, H. Yamaguchi, M. Tanimura, Y. Tagaya, Mechanism and control of cloud cavitation, Journal of Fluids Engineering 119 (4) (1997) $788-794$.

[6] T. M. Pham, F. Larrarte, D. H. Fruman, Investigation of unsteady sheet cavitation and cloud cavitation mechanisms, Journal of Fluids Engineering 121 (1999) 289-296.

[7] M. Callenaere, J.-P. Franc, J.-M. Michel, M. . Riondet, The cavitation instability induced by the development of a re-entrant jet, Journal of Fluid Mechanics 444 (2001) 223-256.

[8] G. Reisman, Y.-C. Wang, C. Brennen, Observations of shock waves in cloud cavitation, Journal of Fluid Mechanics 355 (1998) 255-283. 
[9] R. Arndt, C. Song, M. Kjeldsen, J. He, A. Keller, Instability of partial cavitation: A numerical/experimental approach, in: 23rd Symposium on Naval Hydrodynamics, Val de Reuil, France, 2000.

[10] J.-B. Leroux, J. Astolfi, J.-Y. Billard, An experimental study of unsteady partial cavitation, Journal of Fluids Engineering 126 (2004) 94-101.

[11] J.-B. Leroux, O. Coutier-Delgosha, J. Astolfi, A joint experimental and numerical study of mechanism associated to instability of partial cavitation on two-dimensional hydrofoil, Physics of Fluids 17 (2005) 052101.

[12] Y. Saito, K. Sato, Bubble collapse propagation and pressure wave at periodic cloud cavitation, in: 6th International Conference on Multiphase Flow, Leipzig, Germany, 2007.

[13] K. Sato, Y. Taguchi, S. Hayashi, High speed observation of periodic cavity behavior in a convergent-divergent nozzle for cavitating water jet, Journal of Flow Control, Measurement \& Visualization 1 (2013) 102-107.

[14] C. Stanley, T. Barber, B. Milton, G. Rosengarten, Periodic cavitation shedding in a cylindrical orifice, Experiments in Fluids 51 (2011) 1189-1200.

[15] C. Stanley, T. Barber, G. Rosengarden, Re-entrant jet mechanism for periodic cavitation shedding in a cylindrical orifice, International Journal of Heat and Fluid Flow 50 (2014) 169-176.

[16] H. Ganesh, Bubbly shock propagation as a cause of sheet to cloud transition of partial cavitation and stationary cavitation bubbles forming on a delta wing vortex, Ph.D. thesis, the University of Michigan (2015). 
[17] B. Stutz, J. Reboud, Two-phase flow structure of sheet cavitation, Physics of Fluids 9 (12) (1997) 3678-3686.

[18] V. Aeschlimann, S. Barre, H. Djeridi, Unsteady cavitation analysis using phase averaging and conditional approaches in a 2D Venturi, Open Journal of Fluid Dynamics 3 (2013) 171-183.

[19] O. Coutier-Delgosha, J. Reboud, Y. Delannoy, Numerical simulation of the unsteady behaviour of cavitating flow, Int. Journal for Numerical Methods in Fluids 42 (2003) 527-548.

[20] B. Shin, Y. Iwata, T. Ikohagi, Numerical simulation of unsteady cavitating flows using a homogeneous equilibrium model, Computational Mechanics 30 (2003) 388-395.

[21] I. Senocak, W. Shyy, Interfacial dynamics-based modelling of turbulent cavitating flow, part 2: Time-dependent computations, International journal for numerical methods in fluids 44 (2004) 997-1016.

[22] Y. Chen, C. Lu, L. Wu, Modelling and computation of unsteady turbulent cavitation flows, Journal of Hydrodynamics 18 (5) (2006) 559-566.

[23] E. Goncalves, Numerical study of unsteady turbulent cavitating flows, European Journal of Mechanics B/Fluids 30 (1) (2011) 26-40.

[24] S. LeMartelot, B. Nkonga, R. Saurel, Liquid and liquid-gas flow at all speeds, Journal of Computational Physics 255 (2013) 53-82.

[25] E. Goncalves, Numerical study of expansion tube problems: Toward the simulation of cavitation, Computers \& Fluids 72 (2013) 1-19. 
[26] E. Goncalves, B. Charriere, Modelling for isothermal cavitation with a fourequation model, International Journal of Multiphase Flow 59 (2014) 54-72.

[27] G. Wallis, One-dimensional two-phase flow, McGraw-Hill, New York (1967).

[28] Y. Delannoy, J. Kueny, Two phase flow approach in unsteady cavitation modelling, in: Cavitation and Multiphase Flow Forum, ASME-FED, vol. 98, pp.153$158,1990$.

[29] E. Goncalves, R. F. Patella, Numerical simulation of cavitating flows with homogeneous models, Computers \& Fluids 38 (9) (2009) 1682-1696.

[30] B. Charriere, J. Decaix, E. Goncalves, A comparative study of cavitation models in a venturi flow, European Journal of Mechanics - B/Fluids 49 (2015) 287-297.

[31] B. Smith, The $k-k l$ turbulence model and wall layer model for compressible flows, in: AIAA 90-1483, $21^{\text {st }}$ Fluid and Plasma Dynamics Conference, Seattle, Washington, 1990.

[32] J.-L. Reboud, B. Stutz, O. Coutier, Two-phase flow structure of cavitation: experiment and modelling of unsteady effects, in: $3^{\text {rd }}$ International Symposium on Cavitation CAV1998, Grenoble, France, 1998.

[33] J. Decaix, E. Goncalves, Time-dependent simulation of cavitating flow with $k-\ell$ turbulence models, Int. Journal for Numerical Methods in Fluids 68 (2012) 1053-1072.

[34] E. Goncalves, J. Decaix, Wall model and mesh influence study for partial cavities, European Journal of Mechanics B/Fluids 31 (1) (2012) 12-29. 
[35] J. Viegas, M. Rubesin, Wall-function boundary conditions in the solution of the Navier-Stokes equations for complex compressible flows, in: AIAA 83-1694, $16^{\text {th }}$ Fluid and Plasma Dynamics Conference, Danver, Massachussetts, 1983.

[36] A. Jameson, W. Schmidt, E. Turkel, Numerical solution of the Euler equations by finite volume methods using Runge-Kutta time stepping schemes, in: AIAA Paper 81-1259, 1981.

[37] P. Roe, Approximate Riemann solvers, parameters vectors, and difference schemes, Journal of Computational Physics 43 (1981) 357-372.

[38] J. Decaix, E. Goncalves, Investigation of three-dimensional effects on a cavitating venturi flow, International of Heat and Fluid Flow 44 (2013) 576-595. 
Table 1: Results of DFT of pressure signals at stations 1 to 3 .

\begin{tabular}{ccccc}
\hline \multicolumn{5}{c}{ frequency $(\mathrm{Hz})$} \\
\hline station & $c_{\text {sinus }}=0.47 \mathrm{~m} / \mathrm{s}$ & $c_{\text {sinus }}=0.92 \mathrm{~m} / \mathrm{s}$ & $c_{\text {sinus }}=1.36 \mathrm{~m} / \mathrm{s}$ & stiffened gas \\
\hline 1 & 24.15 & 39.82 & 42.75 & 40.75 \\
2 & 24.13 & 36.20 & 42.24 & 40.94 \\
3 & 24.13 & 36.20 & 42.23 & 25.74 \\
\hline
\end{tabular}




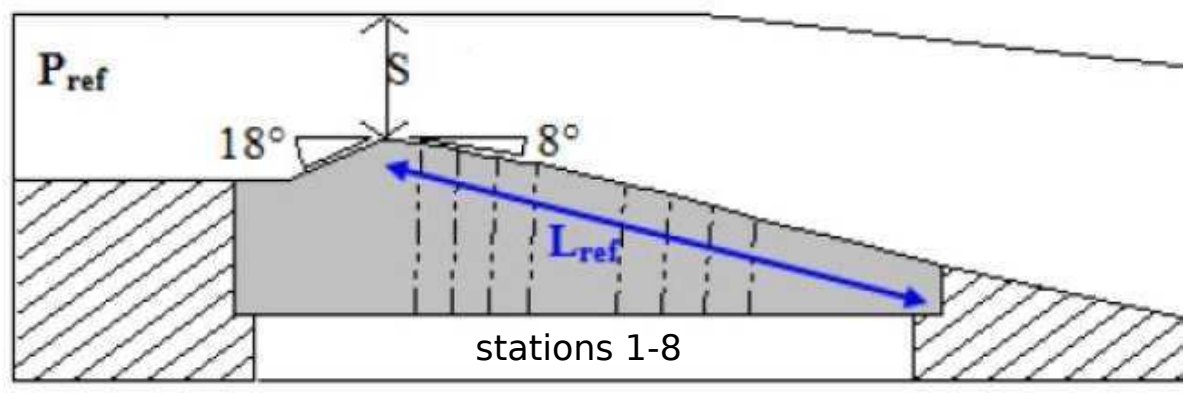

Figure 1: Schematic view of the $8^{\circ}$ Venturi profile with probes location. 


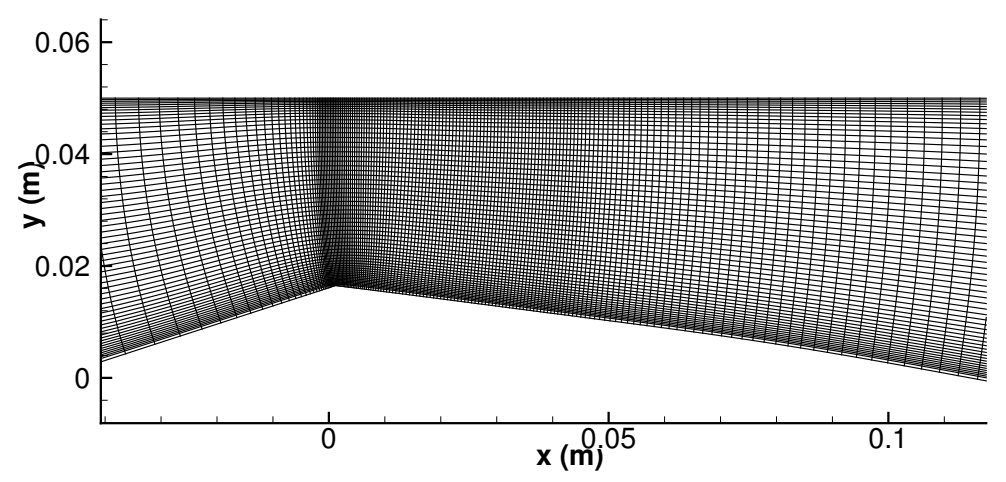

Figure 2: View of the mesh near the Venturi throat. 


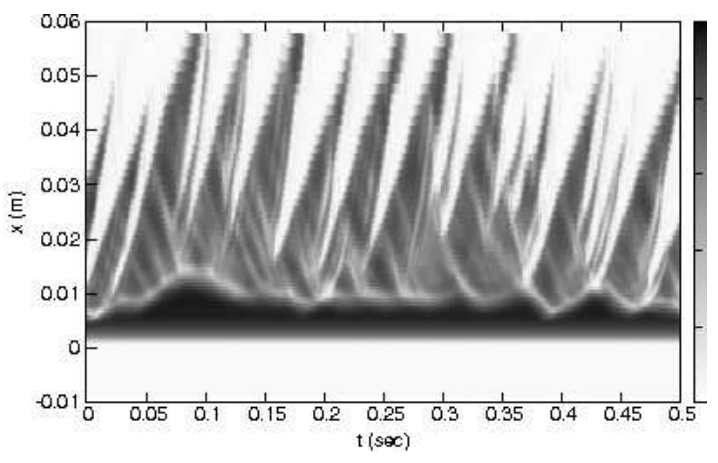

(a) $c_{\text {sinus }}=0.47 \mathrm{~m} / \mathrm{s}$

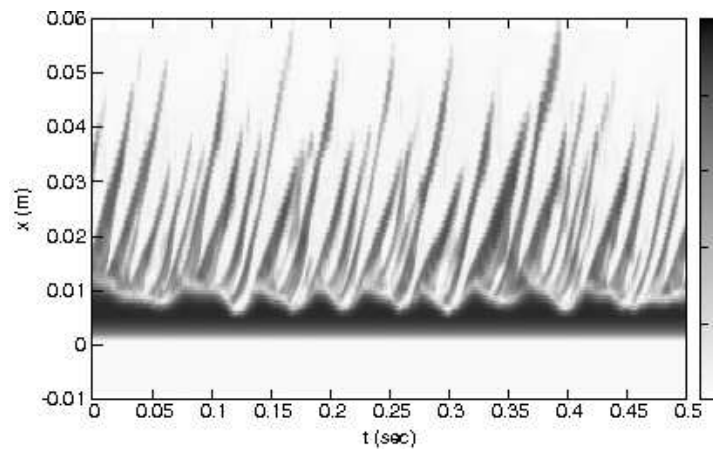

(c) $c_{\text {sinus }}=1.36 \mathrm{~m} / \mathrm{s}$

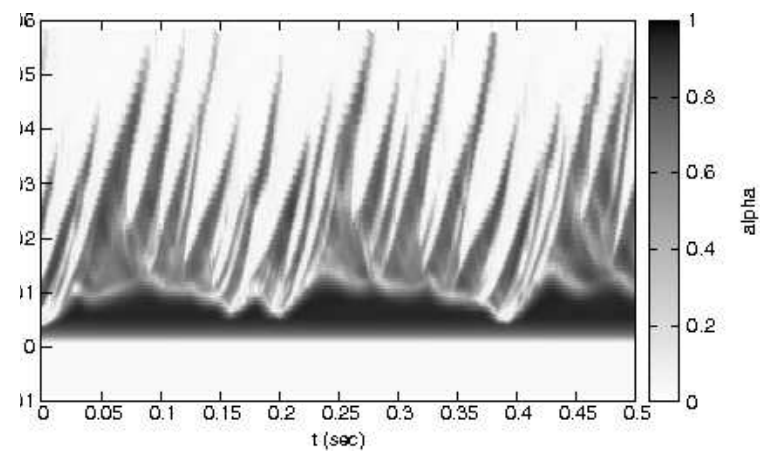

(b) $c_{\text {sinus }}=0.92 \mathrm{~m} / \mathrm{s}$

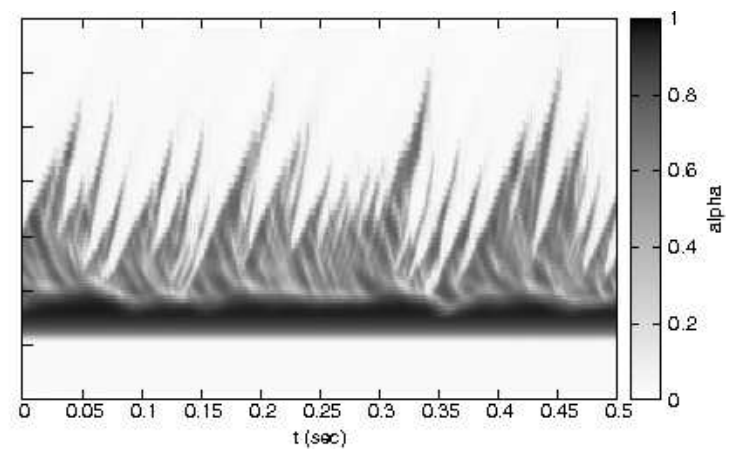

(d) stiffened gas EOS

Figure 3: Maximum void ratio evolution plotted in a spatio-temporal diagram $(x, t)$. The Venturi throat is located at the abscissa $x=0.0012 \mathrm{~m}$. Sub-figures a), b), c) presents the influence of the parameter $c_{\text {sinus }}$ and sub-figure d) the mixture of stiffened gas result. 

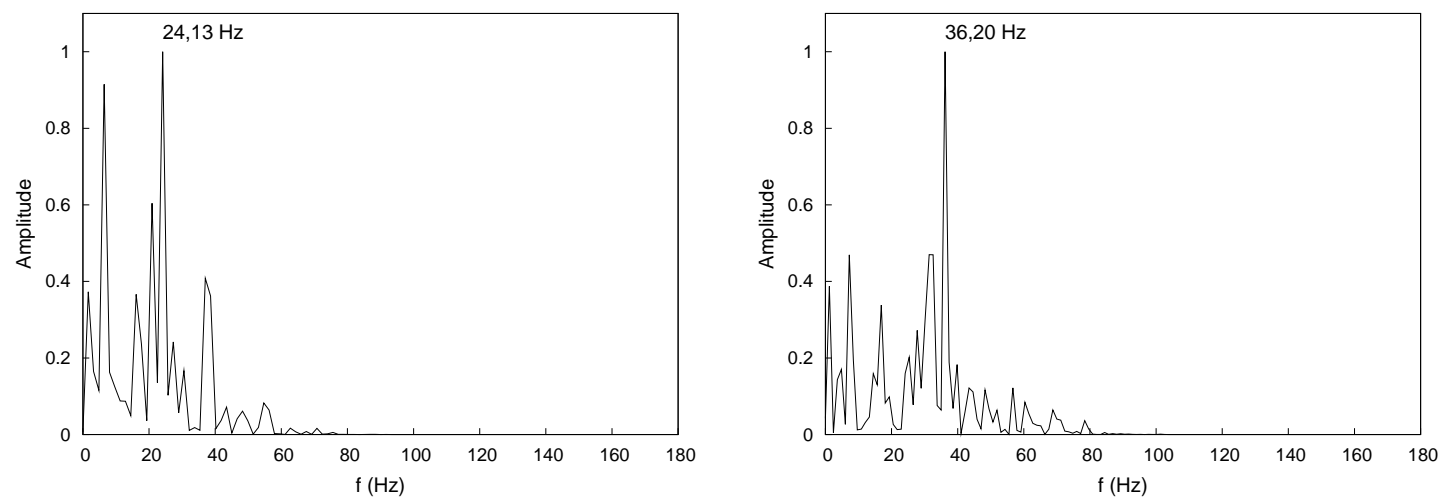

(a) $c_{\text {sinus }}=0.47 \mathrm{~m} / \mathrm{s}$

(b) $c_{\text {sinus }}=0.92 \mathrm{~m} / \mathrm{s}$
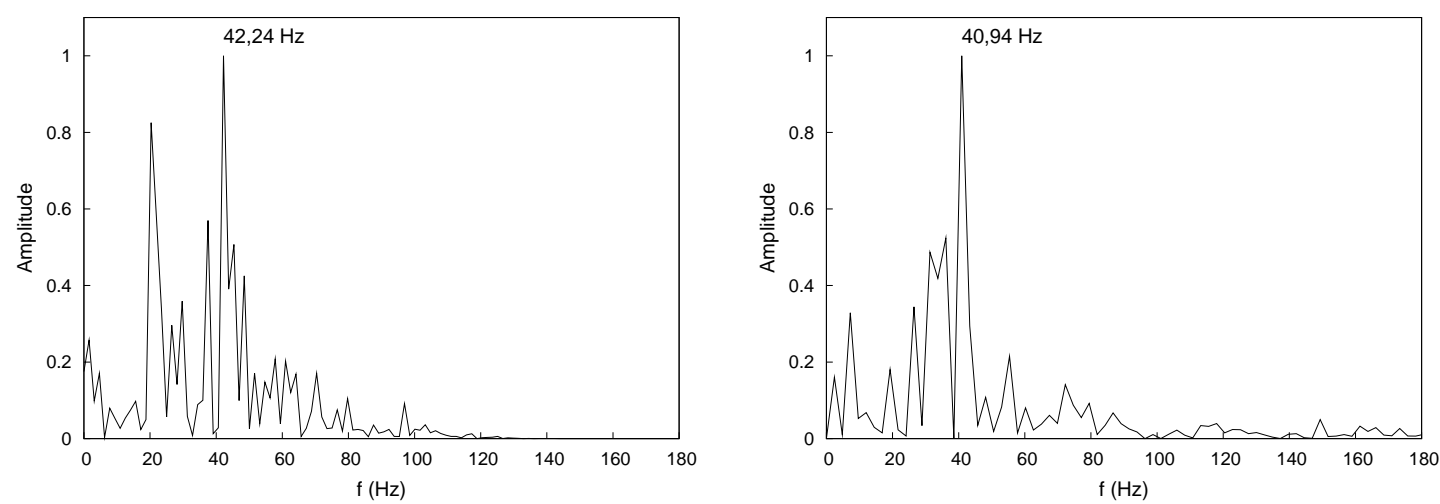

(c) $c_{\text {sinus }}=1.36 \mathrm{~m} / \mathrm{s}$

(d) stiffened gas EOS

Figure 4: Periodic shedding spectra evaluated in the collapse region at station 2 ( $x=20.9 \mathrm{~mm}$ from the throat). Sub-figures a), b), c) presents the influence of the parameter $c_{\text {sinus }}$ and sub-figure d) the mixture of stiffened gas result. 


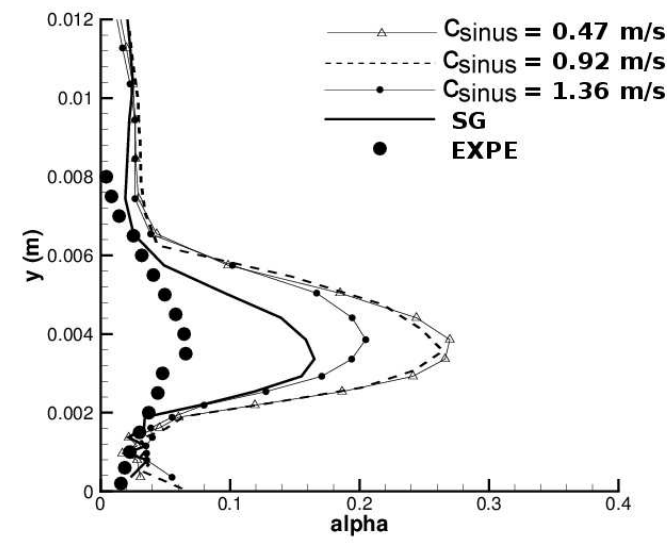

(a) void ratio

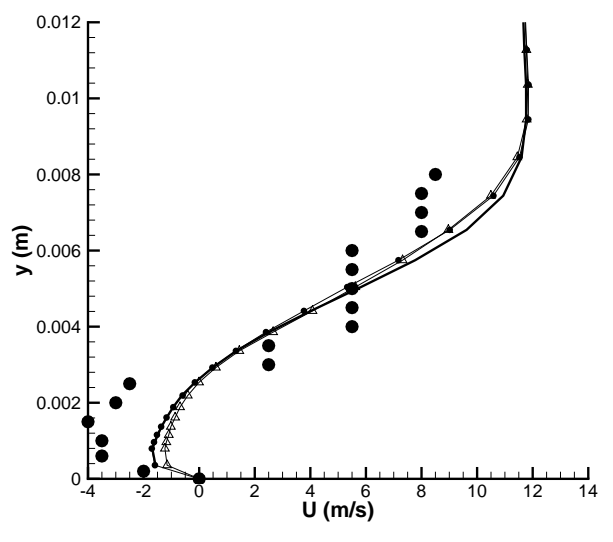

(b) velocity

Figure 5: Comparison of time-averaged void ratio (left) and velocity profiles (right) at station 2, sinusoidal law and stiffened gas (SG) EOS. 

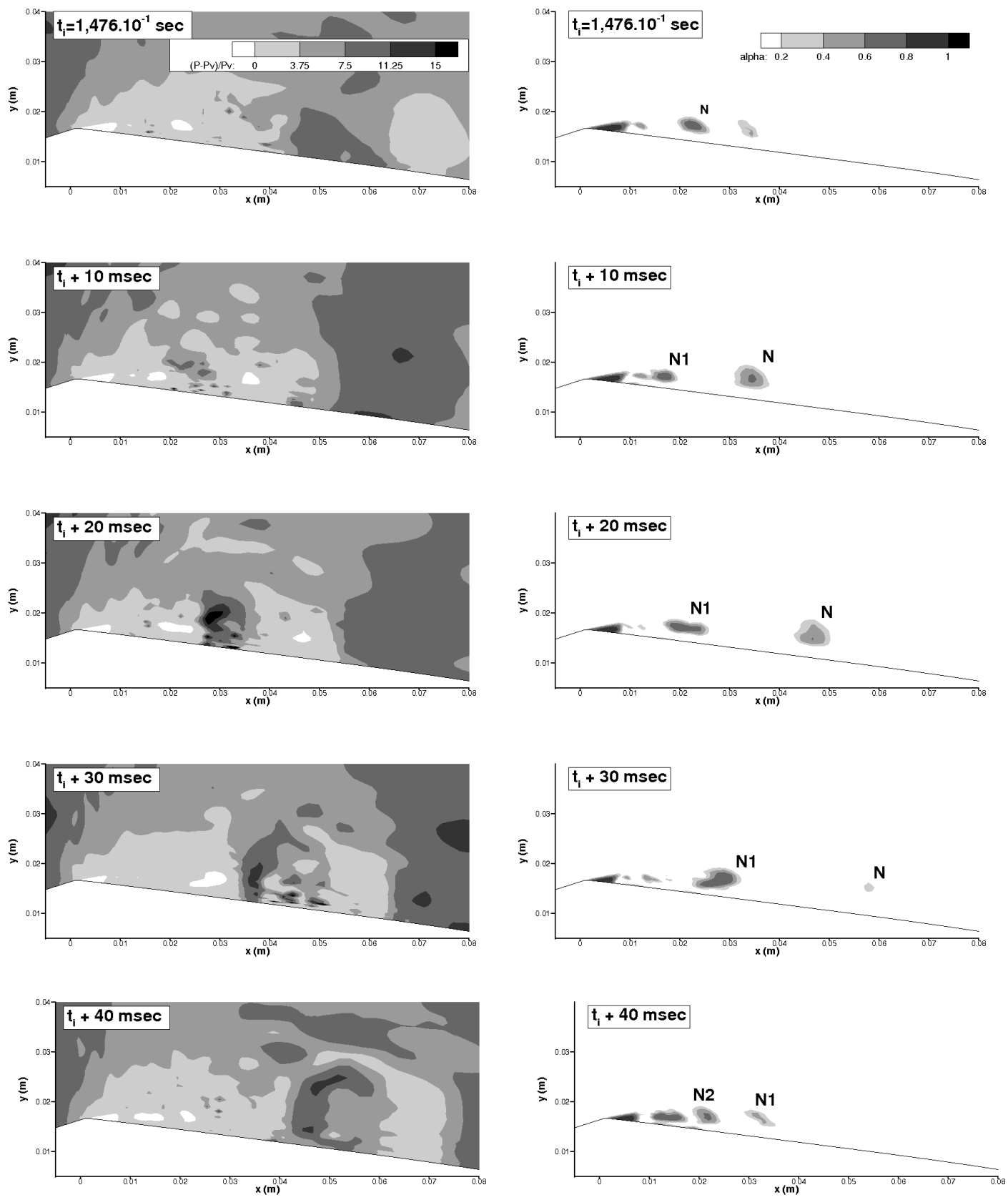

(a) Pressure visualization

(b) Void ratio visualization

Figure 6: Visualization of the pocket dynamics. Instantaneous view of the pressure and void ratio fields at different instants. The time interval between two snapshots is around $10 \mathrm{~ms}$ and the time of observation is $40 \mathrm{~ms}$. Sinusoidal EOS $c_{\text {sinus }} 32.36 \mathrm{~m} / \mathrm{s}$. 

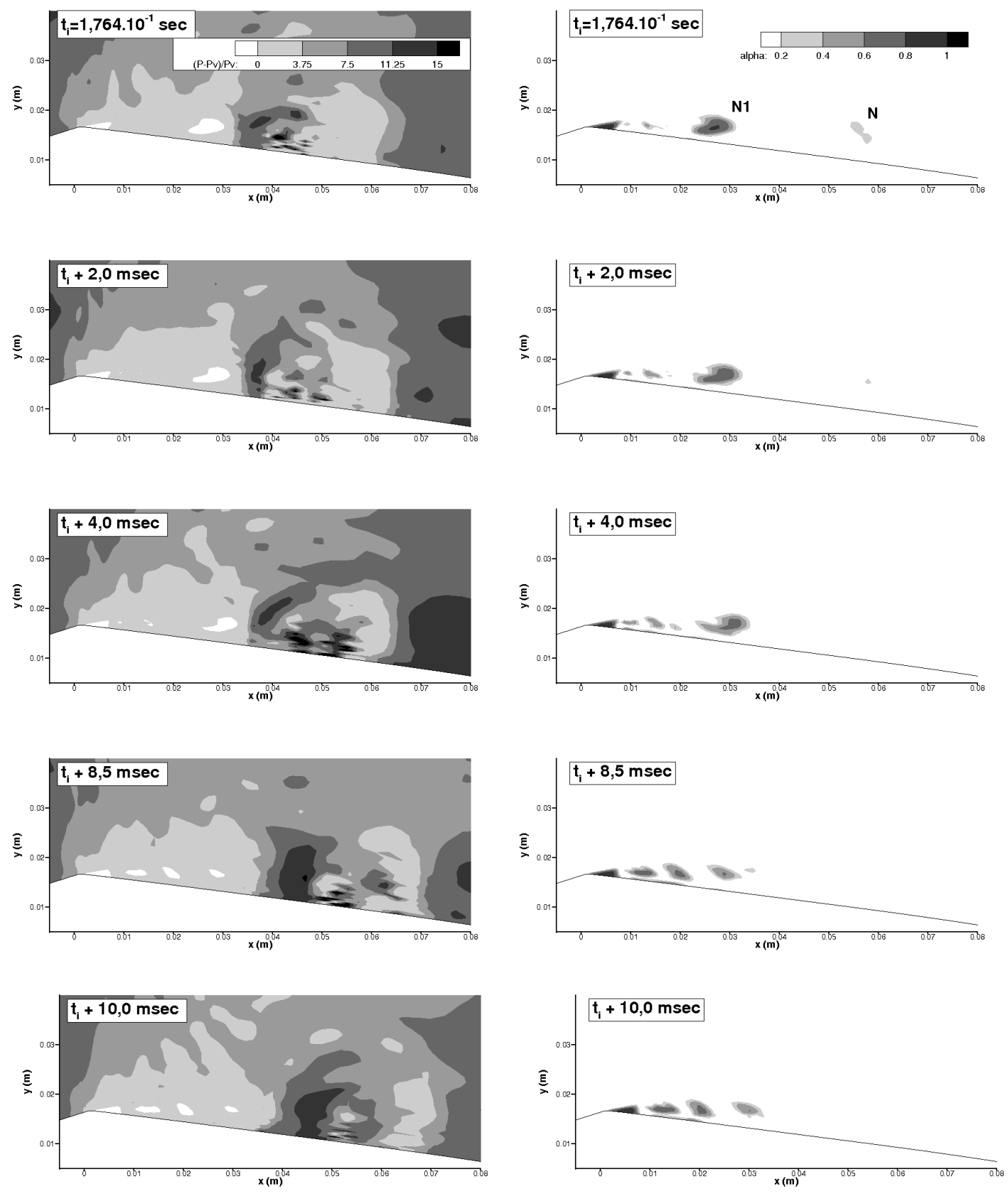

(a) Pressure visualization

(b) Void ratio visualization

Figure 7: Visualization of the pocket dynamics. Instantaneous view of the pressure and void ratio fields at different instants. The time between two snapshots is around $2 \mathrm{~ms}$ and the time of observation is $10 \mathrm{~ms}$. Sinusoidal EOS $c_{\text {sinus }}=1336 \mathrm{~m} / \mathrm{s}$. 

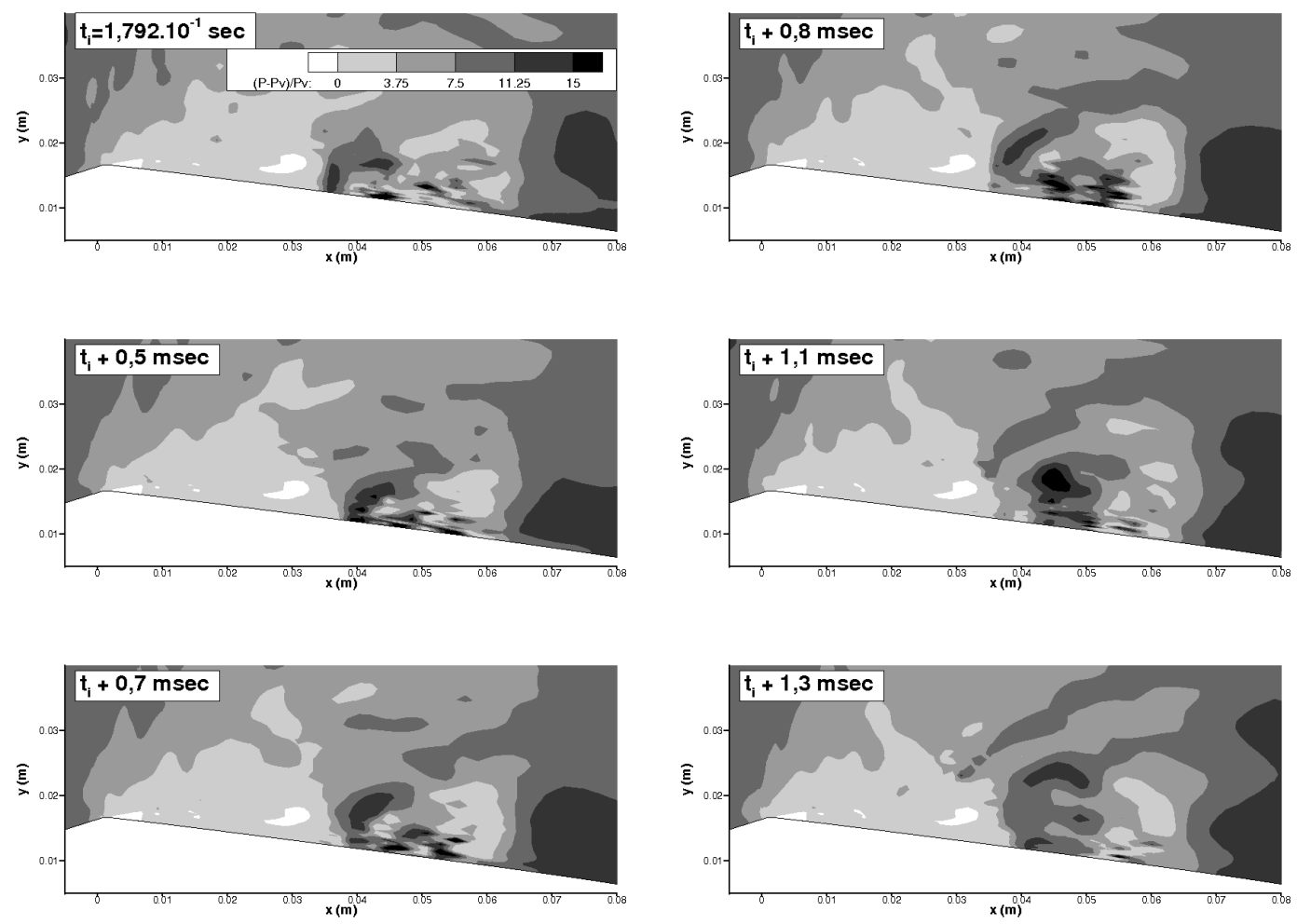

Figure 8: Visualization of the pocket dynamics. Instantaneous view of the pressure field at different instants. The time between two snapshots is around $0.2 \mathrm{~ms}$ and the time of observation is $1.3 \mathrm{~ms}$. Sinusoidal EOS $c_{\text {sinus }}=1.36 \mathrm{~m} / \mathrm{s}$. 


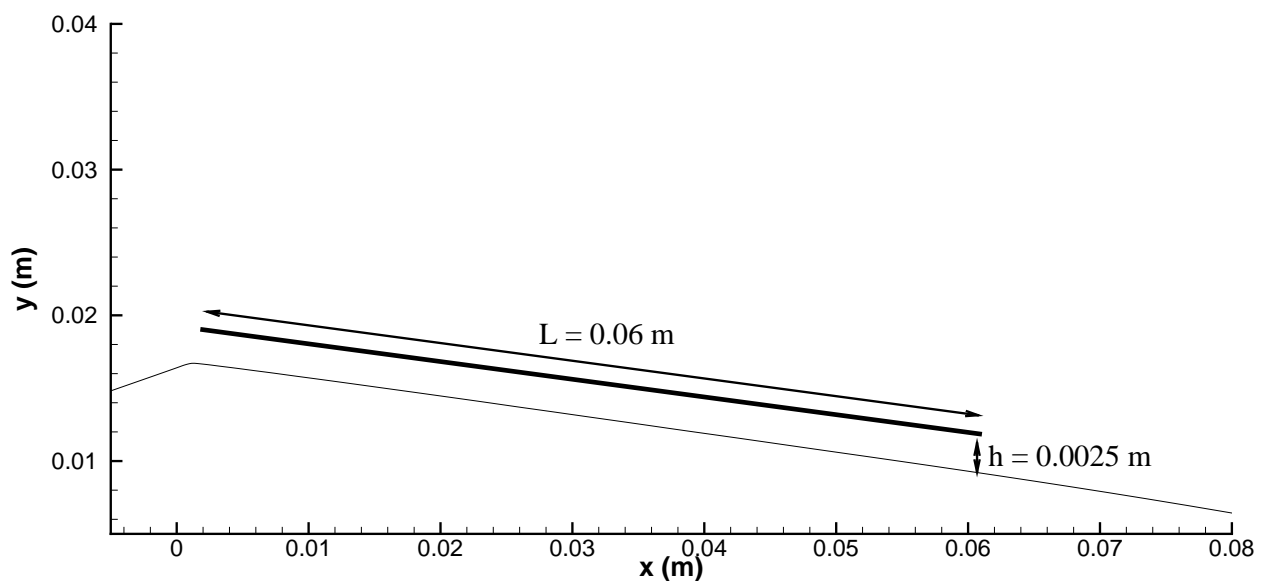

Figure 9: Extraction line for the computation of correlations. 

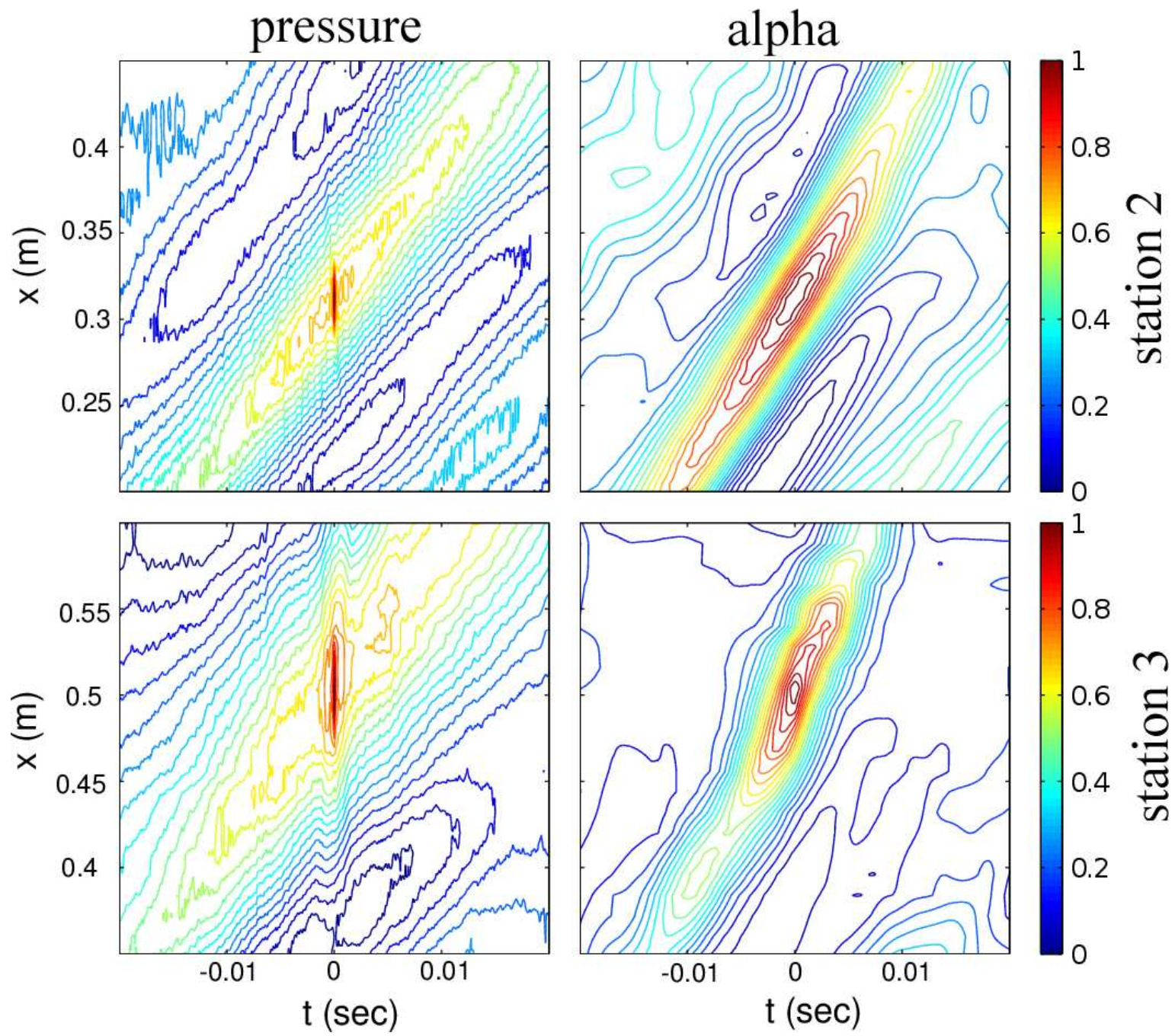

Figure 10: Pressure and void ratio correlations at stations 2 and 3. Sinusoidal EOS $c_{\text {sinus }}=1.36$ $\mathrm{m} / \mathrm{s}$. 

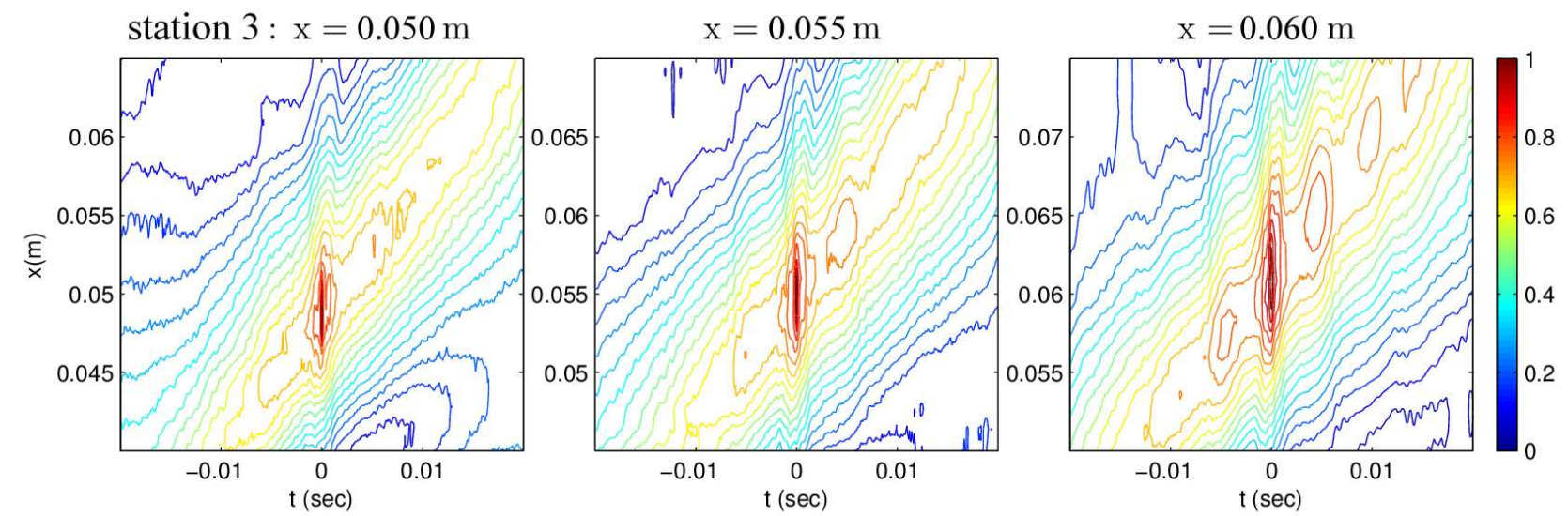

Figure 11: Pressure correlations at station 3 (abscissa $x=0.05 \mathrm{~m}$ ) and downstream. Identification of a fast phenomenon. Sinusoidal EOS $c_{\text {sinus }}=1.36 \mathrm{~m} / \mathrm{s}$. 

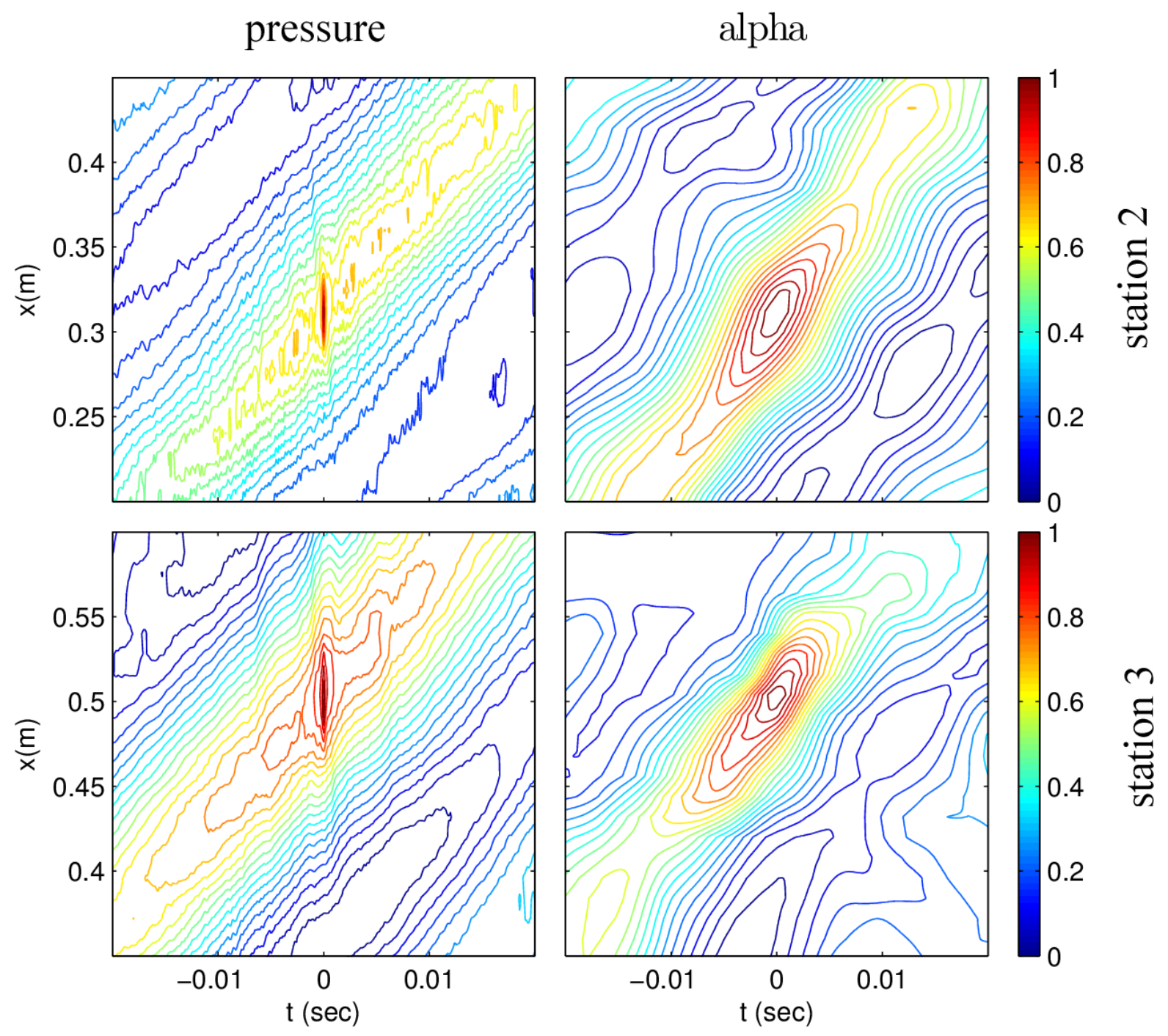

Figure 12: Pressure and void ratio correlations at stations 2 and 3. Sinusoidal EOS $c_{\text {sinus }}=0.47$ $\mathrm{m} / \mathrm{s}$. 


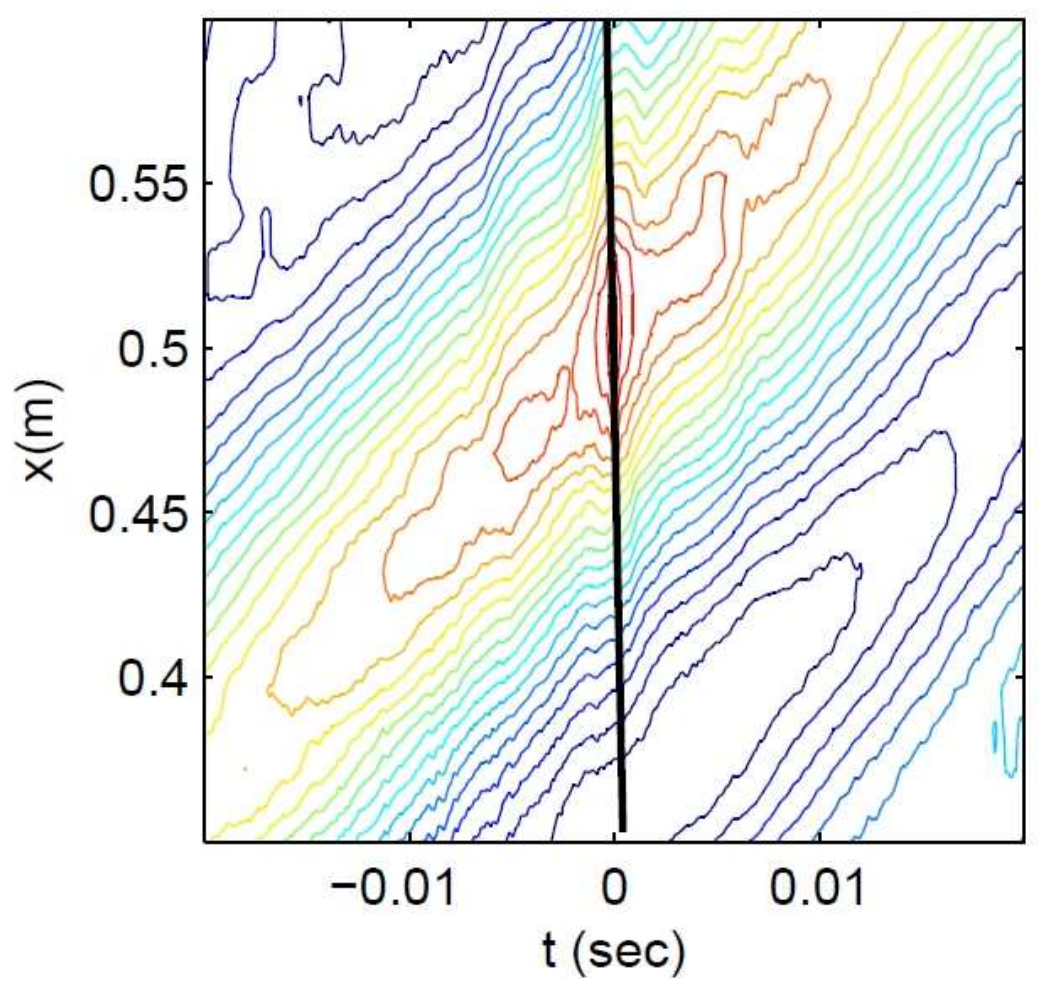

Figure 13: Enlargement of pressure correlations at stations 3. The slope of the black line gives the wave celerity. Sinusoidal EOS $c_{\text {sinus }}=0.47 \mathrm{~m} / \mathrm{s}$. 


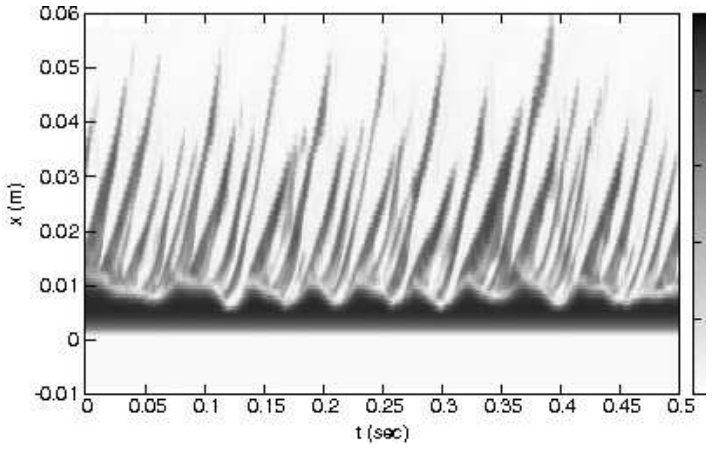

(a) metastable state

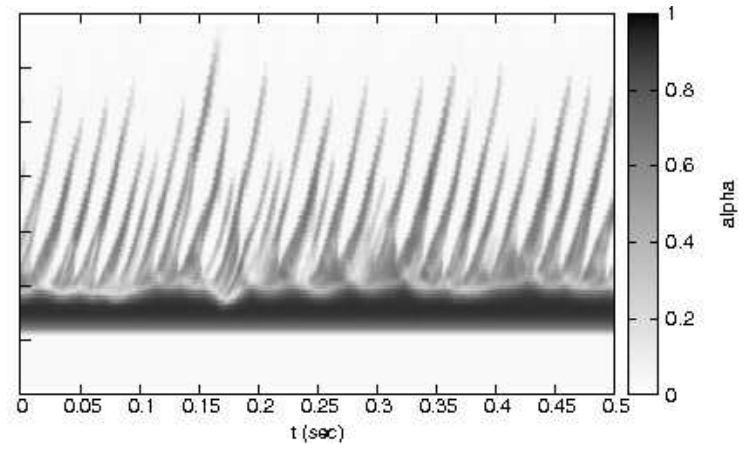

(b) saturation state

Figure 14: Maximum void ratio evolution over time, saturation versus non-equilibrium states. Sinusoidal EOS $c_{\text {sinus }}=1.36 \mathrm{~m} / \mathrm{s}$. 

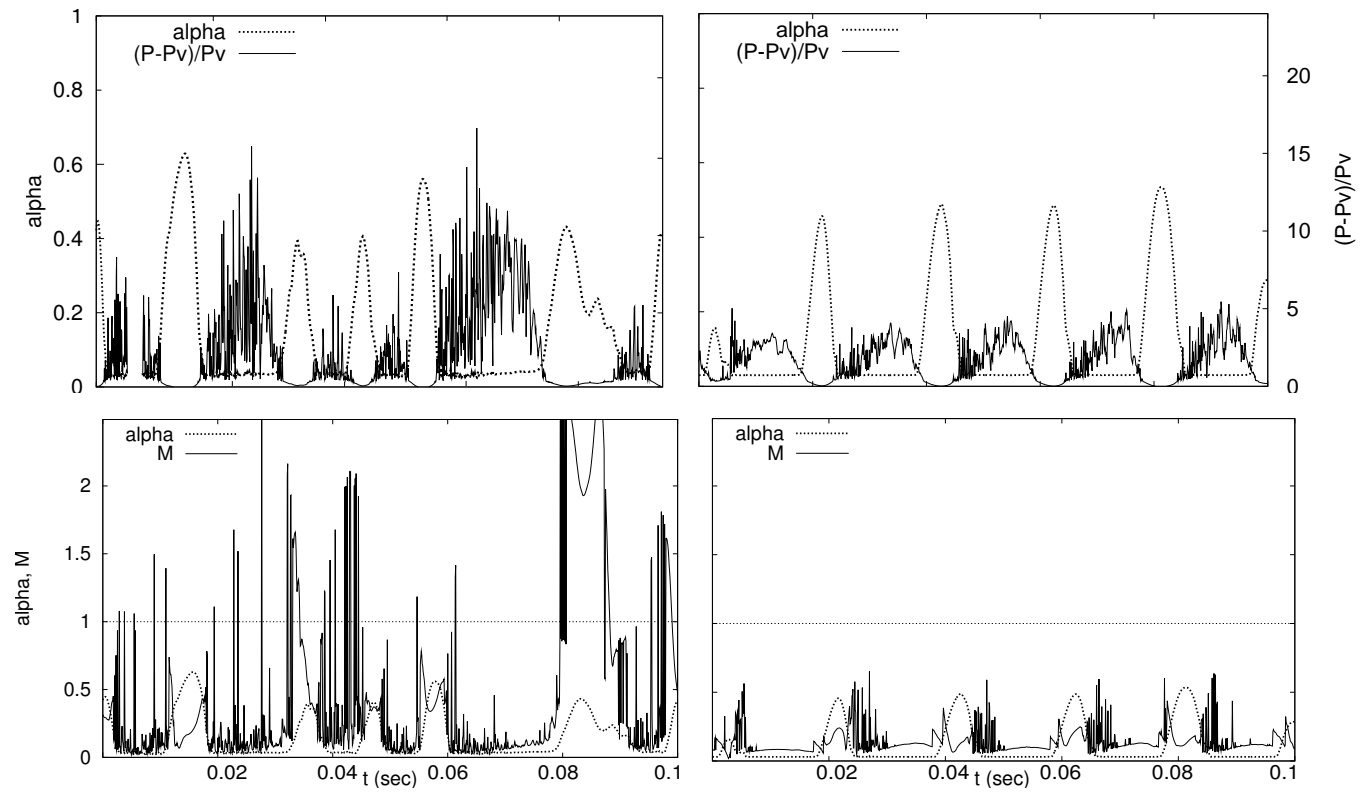

(a) metastable state

(b) saturation state

Figure 15: Temporal evolutions of void ratio, dimensionless pressure and Mach number at station 2 , saturation versus non-equilibrium states. Sinusoidal EOS $c_{\text {sinus }}=1.36 \mathrm{~m} / \mathrm{s}$. 

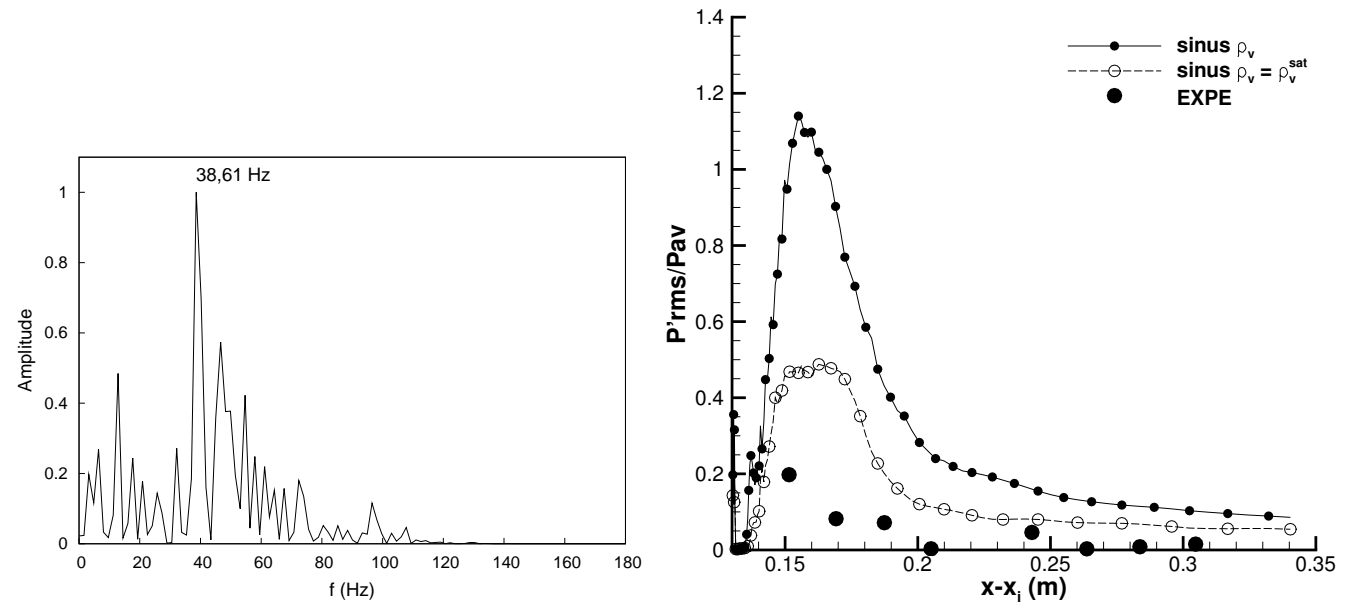

Figure 16: Periodic shedding spectrum evaluated at station 2 (left)and wall RMS pressure fluctuations in the Venturi divergent (right). Sinusoidal EOS $c_{\text {sinus }}=1.36 \mathrm{~m} / \mathrm{s}$. 